\title{
Unruptured aneurysm of the left sinus of Valsalva extending into the left ventricular outflow tract: presentation and imaging
}

\author{
C Pepper, C Munsch, U M Sivananthan, M Pye
}

Department of Cardiology, York District Hospital, Wigginton Road, York YO3 7 HE, UK

C Pepper

M Pye

Department of Cardiothoracic Surgery, Yorkshire Heart Centre, Leeds General Infirmary, Great George Street, Leeds LS1 3EX, UK C Munsch

Department of Radiology, Yorkshire Heart Centre U M Sivananthan

Correspondence to: Dr C Pepper, Department of Cardiology, G Floor, Yorkshire Heart Centre, Leeds General Infirmary, Great George Street, Leeds LS1 3EX, UK.

Accepted for publication 15 December 1997

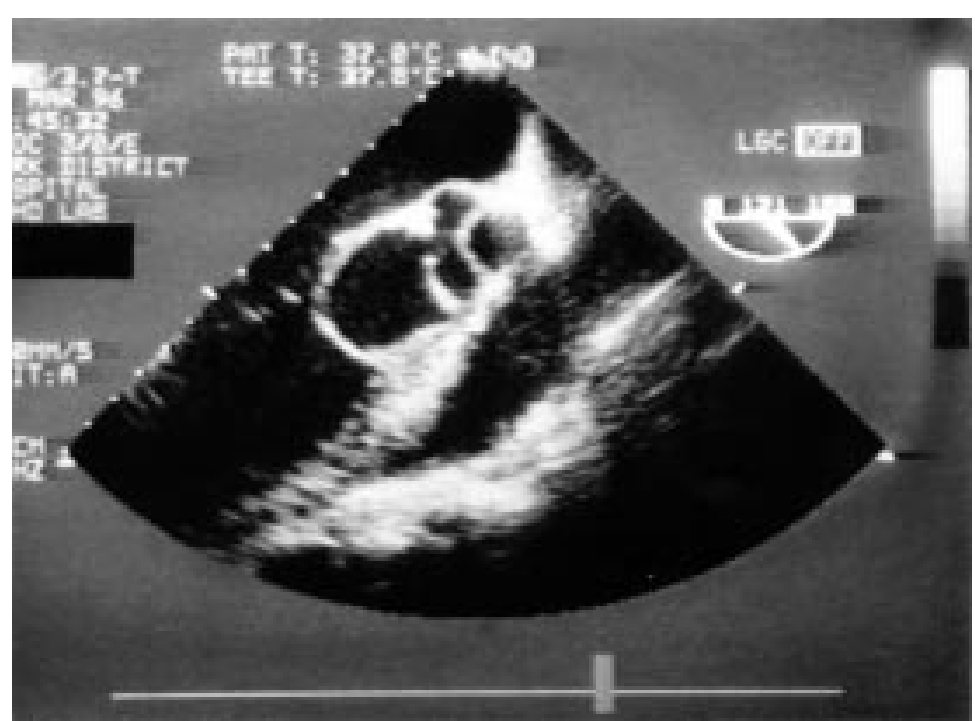

Figure 1 Transoesophageal echocardiogram demonstrating the sinus of Valsalva aneurysm. In this diastolic frame a thin walled saccular structure is seen in communication with the left coronary cusp of the aortic valve.

\section{Abstract}

The symptomatic presentation of an unruptured sinus of Valsalva aneurysm is rare. A 48 year old man with a history of treated hypothyroidism, and a five year history of ileocolonic Crohn's disease of chronic low grade activity presented with a profound left hemiplegia. He was in sinus rhythm and normotensive. Cardiac auscultation was repeatedly normal. Computed tomography of the head performed early in the course of the illness was reported as normal. Duplex Doppler examination of the carotid arteries performed six months later revealed no significant atheroma. There was complete resolution of the neurological deficit over a period of months. A year later he presented with chest pain suggestive of myocardial ischaemia. Computed tomography, magnetic resonance imaging, transthoracic and transoesophageal echocardiography, and cardiac catheterisation pointed to a sinus of Valsalva aneurysm protruding into the left ventricular outflow tract. In view of the previous neurological event and ongoing chest pain

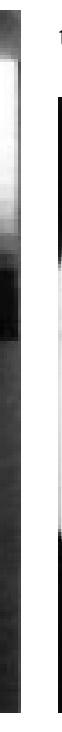
(1)

(1)

suggestive of myocardial ischaemia, the lesion was resected. The patient made a good recovery and postoperative transoesophageal echocardiography showed normal aortic valve function with no residual regurgitation. This is the first reported case of pure left ventricular outflow tract extension of an unruptured left sinus aneurysm. The presentation with ischaemic cardiac pain does not seem to be explained by conventional mechanisms.

(Heart 1998;80:190-193)

Keywords: sinus of Valsalva aneurysm; non-invasive imaging; transoesophageal echocardiography

Aneurysm of the sinus of Valsalva is an uncommon disorder, most commonly presenting subsequent to rupture into a cardiac chamber. Most such aneurysms are thought to be congenital in origin, arising because of discontinuity between the aortic tunica media and aortic valve annulus. ${ }^{1}$ Most unruptured aneurysms are discovered serendipitously, but presentation because of cardiac ischaemia, distal thromboembolism, or arrhythmia has been described. We present a highly unusual form of unruptured sinus of Valsalva aneurysm presenting

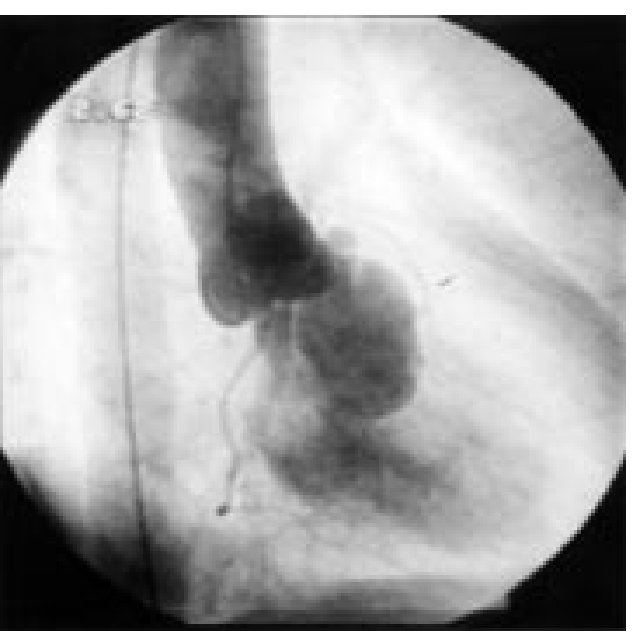

Figure 2 Aortogram (right anterio-oblique projection). e lesion is seen to fill retrogradely from the aortic root in astole. There is mild valvar aortic regurgitation. 


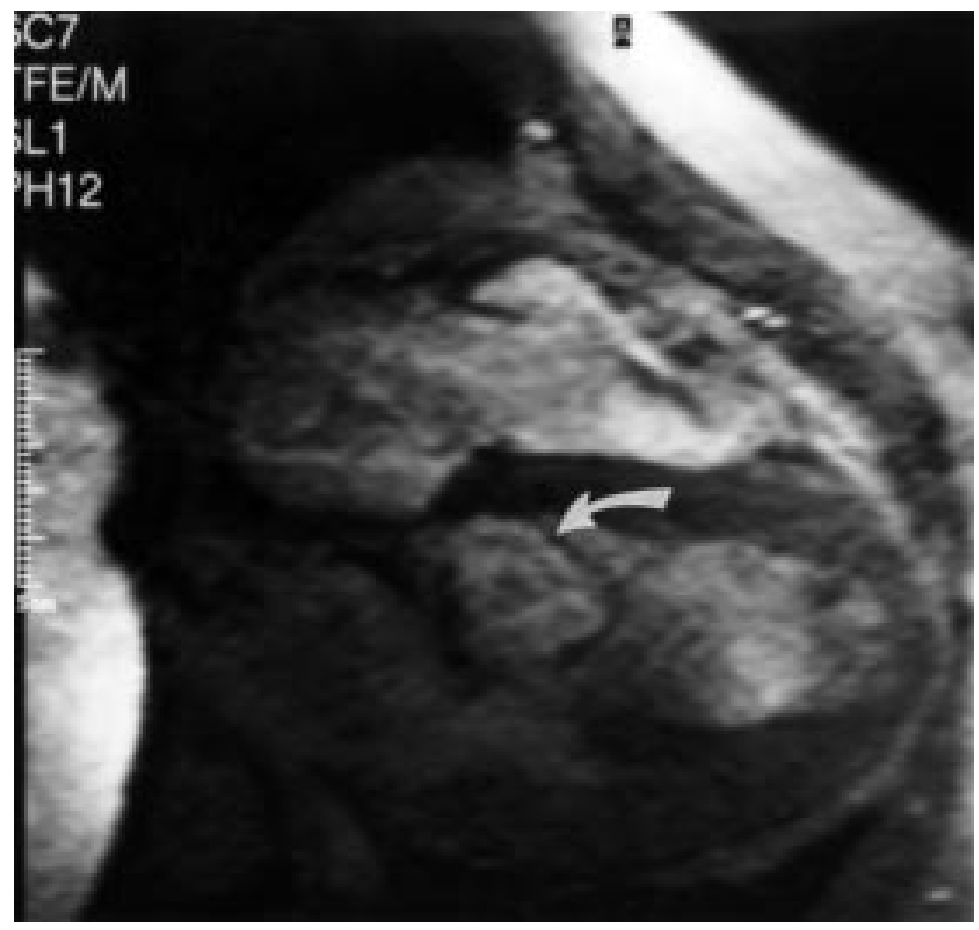

Figure 3 Magnetic resonance imaging showing a low intensity membranous structure within the left ventricular outflow tract (arrow).

with distal thromboembolism and ischaemic cardiac pain, and describe the appearances obtained from a range of imaging techniques.

\section{Case report}

The patient was a 48 year old man with a history of treated hypothyroidism, and a five year history of ileocolonic Crohn's disease of chronic low grade activity. One year before diagnosis he presented with a profound left hemiplegia. He was in sinus rhythm and normotensive. Cardiac auscultation was repeatedly normal. Computed tomography of the head performed early in the course of the illness was reported as normal. Echocardiography was not performed. Duplex Doppler examination of the carotid arteries performed

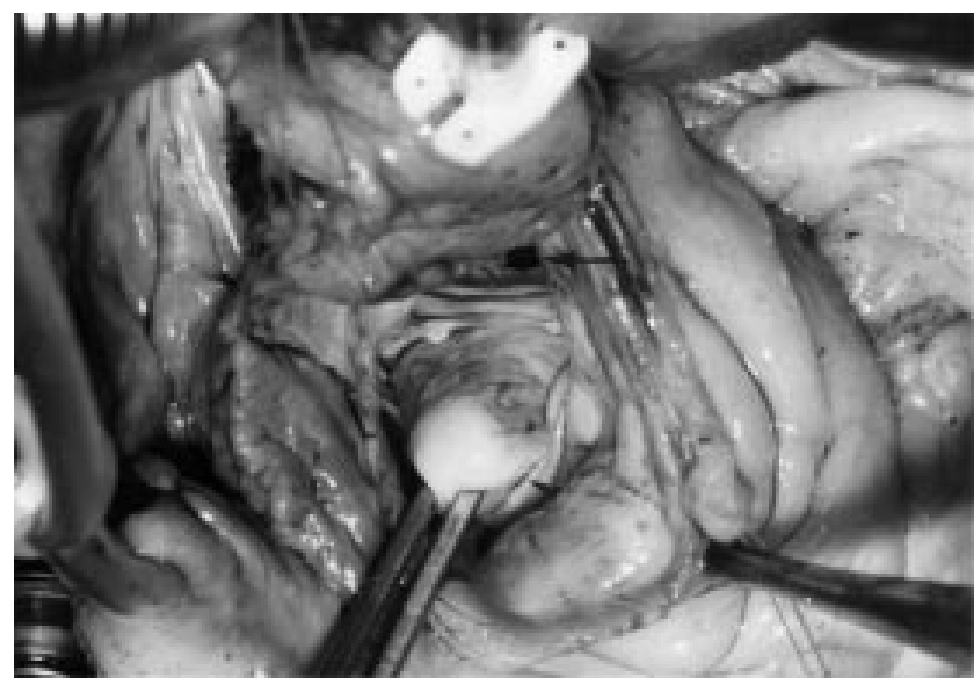

Figure 4 Intraoperative view of the lesion seen from the aortic root. A defect is seen in the left coronary cusp of the aortic valve (arrow). This was in communication with the aneurysm sac, which has been prolapsed through the valve (arrowhead). six months later revealed no significant atheroma. There was complete resolution of the neurological deficit over a period of months.

Twelve months later the patient was referred on an urgent basis. He reported repeated episodes of central chest discomfort over three days, which he described as feeling "like a bag of sand on his chest". The pain was exertional in nature and associated with mild diaphoresis. On examination he was afebrile and in sinus rhythm. The pulse character was normal. A grade $2 / 6$ decrescendo early diastolic murmur was audible over the left sternal edge, with a grade $3 / 6$ ejection systolic murmur in the same area radiating to the carotids. The chest was clear. A 12 lead ECG revealed a partial right bundle branch block and was unchanged from the previous presentation. Chest radiography was normal. Five sets of blood cultures proved sterile; erythrocyte sedimentation rate and C reactive protein concentrations were slightly raised consistent with Crohn's disease of chronic low grade activity.

Transthoracic and transoesophageal echocardiography showed a thin walled saccular lesion in the left ventricular outflow tract (fig 1). The lesion was associated with the aortic valve and interventricular septum, and separate from the mitral valve. There was an associated discontinuity within the left coronary cusp of the aortic valve through which the lesion filled in diastole, subsequently collapsing in systole. No thrombus was seen. There was mild aortic regurgitation around the lesion and turbulent flow was present within it during diastole. The aortic valve cusps were otherwise normal and no vegetations were seen. The mitral valve was normal. The left ventricle was not dilated and systolic function was good.

Contrast aortography and left ventriculography showed the lesion to be entirely within the left ventricular outflow tract and filled retrogradely from above the left coronary sinus (fig 2). Grade 2/4 aortic regurgitation was present but there was no evidence of perforation of the lesion. The coronary arteries were normal.

Because of continuing diagnostic debate, magnetic resonance imaging (MRI) was performed. Axial, four chamber, and short axis spin echo breath hold images were obtained revealing a protruding membrane in the left ventricular outflow tract (fig 3), which prolapsed into the outflow tract in diastole and through the aortic valve in systole. Gradient echo images suggested a small diastolic leak consistent with a small area of perforation.

In view of the previous neurological event and ongoing chest pain suggestive of myocardial ischaemia the lesion was resected. At surgery, the external appearances of the heart were unremarkable. On opening the ascending aorta a $0.5 \mathrm{~cm}$ circular defect was found in the left coronary sinus (fig 4), which communicated with a large left sinus of Valsalva aneurysm. The aneurysm lay completely within the left ventricle and was partially adherent to the posterior ventricular wall and to the subvalvar apparatus of the mitral valve. 
The aortic valve was functionally and structurally normal. The aneurysm was excised completely and the defect in the left coronary sinus closed with a patch of gluteraldehyde treated bovine pericardium. The patient made a good recovery and postoperative transoesophageal echocardiography showed normal aortic valve function with no residual regurgitation.

\section{Discussion}

Although the rate of discovery has increased with the widespread availability of echocardiography, unruptured sinus of Valsalva aneurysms remain very uncommon. The appearances of the present lesion were highly unusual. Most sinus of Valsalva aneurysms arise from the right and non-coronary sinuses, only $1-2 \%$ arising from the left. ${ }^{2}$ They typically occur above the aortic valve ring and may extend in any direction. Aneurysms of right or noncoronary cusp origin commonly involve the right atrium and right ventricular outflow tract, and lesions from all three sinuses may occasionally compress or stretch a coronary artery (below). Several cases have been reported where a right coronary sinus aneurysm has extended into the interventricular septum, ${ }^{3-6}$ but saccular extension into the left ventricular outflow tract is very uncommon. To our knowledge only two previous cases showing similar appearances on imaging have been reported, both arising from the right coronary sinus. ${ }^{78}$ Similar appearances from a left sinus aneurysm have not previously been described.

We believe this to be the first report describing the MRI appearances of an unruptured sinus of Valsalva aneurysm. Cardiac MRI has the potential to be of considerable value in the evaluation of these lesions. The ability of this technique to enable the lesion to be viewed in any plane allowed clear delineation of the origin of the lesion, and provided the clear demonstration of regional blood flow. This suggested the presence of a small leak from the aneurysm not seen by other imaging techniques. Such information could potentially affect decisions regarding intervention.

Most unruptured sinus of Valsalva aneurysms are silent, being discovered serendipitously, but a number of modes of presentation have been reported. These include arrhythmias including atrial fibrillation, ${ }^{9}$ ventricular tachycardia, ${ }^{10}$ and complete heart block, ${ }^{3}$ and heart failure resulting from aortic regurgitation ${ }^{7}$ or right ventricular outflow tract obstruction. ${ }^{11}{ }^{12}$ Sudden death has occurred, and has been suggested to result from coronary artery obstruction rather than a primary arrhythmia. ${ }^{13} \mathrm{~A}$ few cases have been reported as presenting with distal thromboembolism, including stroke. ${ }^{14}$

The mechanisms of cardiac ischaemia arising from sinus of Valsalva aneurysms have been reviewed by Brandt et al. ${ }^{5}$ The 16 reported cases in their survey had an excess of left sinus aneurysms. This may reflect reporting bias, but may be a function of the anatomical relation between the left coronary sinus and the proximal left coronary artery. In nine of 10 left sinus aneurysms the chest pain was thought to be caused by direct compression of the left main stem or its branches. In our case, chest pain suggestive of cardiac ischaemia was a presenting feature; the mechanism is not immediately apparent. The coronary arteries were angiographically normal and there was no evidence of distortion by the aneurysm, which was entirely subvalvar. Myocardial ischaemia may occur in pure aortic incompetence caused by an increase in left ventricular end diastolic pressure and consequent reduction in coronary perfusion pressure, but in this case the aortic incompetence was not sufficiently severe and the left ventricular end diastolic pressure was normal at catheterisation. It is possible that diastolic filling of the aneurysm from the left sinus may have provoked a "steal" phenomenon, resulting in reduced left coronary artery flow. Alternatively intermittent prolapse of the aneurysmal membrane through the aortic valve could have resulted in intermittent coronary ostial obstruction.

One year before the index presentation the patient presented with an acute left hemiplegia suggestive of an acute cerebrovascular event. Cranial computed tomography showed no evidence of haemorrhage, and an embolic event seems likely. Although an echocardiographic examination was not carried out at that time, it seems reasonable to assume the aneurysm was present and is highly likely to be an embolic source.

The optimal management of the unruptured sinus of Valsalva aneurysm is debated. The risk of rupture is impossible to predict as the majority probably remain undiagnosed. While there may a case for the expectant management of asymptomatic aneurysms, ${ }^{15}$ those that lead to symptoms are probably best dealt with aggressively. Rapid expansion has been reported in at least one case, ${ }^{13}$ and there is a clear risk of sudden death in patients with symptoms of myocardial ischaemia. ${ }^{5}$ Aortic incompetence from valvar distortion can be alleviated by aneurysmectomy without the need for valve replacement in most cases. The best treatment of distal thromboembolism is unclear, but surgical intervention obviates the need for prolonged anticoagulation and its attendant complications. For these reasons operative intervention was thought to be the treatment of choice in our case.

In conclusion, we describe the findings on non-invasive imaging of a highly unusual form of sinus of Valsalva aneurysm. We believe to be the first reported case of pure left ventricular outflow tract extension of an unruptured left sinus aneurysm. The presentation with ischaemic cardiac pain does not seem to be explained by conventional mechanisms.

1 Edwards JE, Burchell HB. The pathologic anatomy of deficiencies between the aortic root and the heart including aortic sinus aneurysms. Thorax 1957;12:125-39.

2 Dev V, Goswami KC, Shrivastava S, et al. Echocardiographic diagnosis of aneurysm of the sinus of Valsalva. Am Heart $\mathcal{F}$ 1993;126:930-6.

3 Ahmad RAS, Sturman S, Watson RDS. Unruptured aneurysm of the sinus of Valsalva with isolated heart block: echocardiographic diagnosis and successful surgical repair. Br Heart 7 1989;61:375-7.

Br Heart f 1989;61:375-7.
4 Dev V, Shrivastava S. Echocardiographic diagnosis of unruptured aneurysm of the sinus of Valsalva dissecting into the ventricular septum. Am F Cardiol 1990;66:502-3. 
5 Brandt J, Jögi P, Lührs C. Sinus of Valsalva aneurysm obstructing coronary arterial flow: case report and 73 .

6 Lewis BS, Agathangelou NE. Echocardiographic diagnosis of unruptured sinus of Valsalva aneurysm. Am Heart $\mathcal{F}$ 1984;107:1025-7.

7 Mok CK, Cheung KI, Wang RYC. Unruptured right coronary sinus to left ventricle aneurysm diagnosed by cross sectional echocardiography. Br Heart f 1985;53:2269.

8 Heydorn WH, Nelson WP, Fitterer JD, et al. Congenital aneurysm of the sinus of Valsalva protruding into the left ventricle. F Thorac Cardiovasc Surg 1976;71:839-45.

9 Walsh JT, Andrews R. Unruptured aneurysm of the left sinus of Valsalva presenting with atrial fibrillation. Int $\mathcal{F}$ Cardiol 1994;46:297-8.

10 Channer KS, Hutter JA, George M. Unruptured aneurysm of the sinus of Valsalva presenting with ventricular of the sinus of Valsalva presenting
tachycardia. Eur Heart $\mathcal{7}$ 1988;9:186-90.
11 Kiefaber RW, Tabakin BS, Coffin LH, et al. Unruptured sinus of Valsalva aneurysm with right ventricular outflow obstruction diagnosed by two dimensional and Doppler echocardiography. $\mathcal{F}$ Am Coll Cardiol 1986;7:438-42.

12 Desai AG, Sharma S, Kumar A, et al. Echocardiographic diagnosis of unruptured aneurysm of right sinus of Valsalva: an unusual cause of right ventricular outflow obstruction. Am Heart F 1985;109:363-4.

13 Faillance RT, Greenland P, Nanda NC. Rapid expansion of a saccular aneurysm on the left coronary sinus of Valsalva: a role for early surgical repair? $\mathrm{Br}$ Heart $\mathcal{f}$ 1985;54:442-4.

14 Stölberger C, Seitelberger R, Fenninger C, et al. Aneurysm of the left sinus of Valsalva. An unusual source of cerebral embolism. Stroke 1996;27:1424-6.

15 Rothbart RM, Chahine RA. Left sinus of Valsalva aneurysm with rupture into the left ventricular outflow tract: diagnosis by color-encoded Doppler imaging. Am Heart $\mathcal{f}$ 1990;120:224-7. 